\title{
Avaliação do Efeito de Dica Semântica e da Indução de Significado entre Estímulos Abstratos Equivalentes
}

\author{
Evaluation of Semantic Priming Effect and Induction of Meaning \\ among Abstract Equivalent Stimuli
}

\author{
Renato Bortoloti* \& Julio Cesar Coelho de Rose \\ Universidade Federal de São Carlos, São Carlos, Brasil
}

\begin{abstract}
Resumo
Relações semânticas entre estímulos equivalentes foram avaliadas utilizando "tentativas de decisão léxica" e diferencial semântico. Foram utilizadas as estruturas de treino conhecidas como "um-para-muitos" e "muitos-para-um" na geração de três classes de estímulos equivalentes envolvendo cinco conjuntos de estímulos: A, B, C, D e E. Para um grupo de participantes, denominado de Grupo Um para Muitos (GUM), as relações diretamente treinadas obedeciam à estrutura $\mathrm{AB}, \mathrm{BC}, \mathrm{CD}$ e $\mathrm{CE}$; um outro grupo experimental, denominado de Grupo Muitos para Um (GMU), foi treinado na estrutura DC, EC, CB e BA. Para os dois grupos, os estímulos do conjunto B eram fotografias de faces expressando emoções e os estímulos dos conjuntos A, C, D e E eram figuras abstratas. A seguir, duas figuras (já vistas ou novas) eram apresentadas em seqüência, como "dica" e "alvo", em tentativas em que o participante respondia se reconhecia ou não a figura alvo. As respostas foram mais rápidas quando dica e alvo pertenciam à mesma classe, reproduzindo com estímulos abstratos equivalentes o efeito de dica semântica. Figuras abstratas foram então avaliadas por diferencial semântico. As avaliações do GUM foram similares às avaliações das faces por um grupo controle e as avaliações do GMU não foram. Os dados sugerem que a intensidade relativa da transferência de significados entre estímulos equivalentes pode depender da direção das relações que os participantes devem estabelecer.

Palavras-chave: Equivalência de estímulos; Transferência de significado; Dica semântica; Diferencial semântico.

Abstract

Semantic relations among equivalent stimuli were evaluated by lexical decision tasks and a semantic differential. Two training structures, known as "one-to-many" and "many-to-one", were employed in order to generate three classes of equivalent stimuli involving five sets of stimuli: A, B, C, D and E. Stimuli for set B were pictures of faces expressing emotions whereas stimuli for sets A, C, D and E were abstract pictures. For one group of participants, the One-to-many Group, the directly trained relations were $\mathrm{AB}$, $\mathrm{BC}, \mathrm{CD}$ and $\mathrm{CE}$; for the other experimental group, the Many-to-one Group, the directly trained relations were DC, EC, CB and BA. Two pictures were then presented in sequence as "prime" and "target" in trials in which participants responded whether they recognized or not the target. Responses were faster when prime and target belonged to the same equivalent class, reproducing the semantic priming effect. Abstract pictures were then evaluated through the semantic differential. Evaluations by the One-to-many Group were similar to the evaluations of the faces made by a control group, but those made by the Many-to-one Group were not. The data suggest that the transfer of meaning among equivalent stimuli may depend on the relations which are established by the participants.

Keywords: Stimuli equivalence; Transfer of meaning; Semantic priming; Semantic differential.
\end{abstract}

O modelo de equivalência de estímulos de Sidman e colaboradores (e.g., Bush, Sidman, \& T. de Rose, 1989; Sidman, 1994; Sidman \& Tailby, 1982) deu uma dimen-

\footnotetext{
* Endereço para correspondência: Universidade Federal de São Carlos, Centro de Educação e Ciências Humanas, Departamento de Psicologia, Rodovia Washington Luiz, KM 235, cx. postal 676, Monjolinho, São Carlos, SP, Brasil, CEP 13565-905. E-mails: renatobortoloti@yahoo.com.brejuliocderose@yahoo.com.br
}

são experimental mais ampla a processos de análise comportamental da linguagem humana ao apresentar critérios operacionais para um tratamento empírico de temas como o uso de símbolos. Sidman e Tailby (1982) especificaram, por exemplo, testes precisos que parecem confirmar se as relações comportamentais estudadas atingem ou não as condições para serem consideradas como relações simbólicas. Os autores propuseram que o critério para identificar relações comportamentais simbólicas pode ser derivado daquele proposto pela teoria 
dos conjuntos para identificar relações de equivalência: a demonstração das propriedades de reflexividade, simetria e transitividade. De acordo com essa proposta, o símbolo seria qualquer estímulo relacionado a algo, ou seja, um outro estímulo, por meio de uma relação de equivalência.

A teoria dos conjuntos estabelece que relações de equivalência devem ter as propriedades necessárias para conferir substitutabilidade recíproca aos elementos envolvidos. Assim, se um elemento A é relacionado de uma maneira particular a um elemento B e se esse elemento B é relacionado da mesma maneira a um elemento $C$, os elementos $\mathrm{A}, \mathrm{B}$ e C serão equivalentes se a relação entre eles for, ao mesmo tempo, transitiva, simétrica e reflexiva. Em outras palavras, se A é relacionado a $\mathrm{B}$ e B é relacionado a $C$, então, para que $A, B$ e $C$ sejam equivalentes, A e C, C e A, B e A, C e B, A e ele mesmo, B e ele mesmo e $\mathrm{C}$ e ele mesmo também deverão estar relacionados da mesma maneira. $\mathrm{O}$ modelo de equivalência de estímulos propôs indicadores comportamentais derivados das propriedades lógicas das relações de equivalência para verificar se uma dada relação entre estímulos tem características simbólicas. Se essa relação puder ser caracterizada como uma relação de equivalência, definida pelas propriedades de reflexividade, simetria e transitividade, os estímulos relacionados formarão uma classe de estímulos equivalentes e a relação entre eles será simbólica.

A especificação operacional oferecida pelo modelo de equivalência permitiu simular função simbólica em laboratório. Nessas simulações, são utilizados procedimentos de emparelhamento cujas características principais podem ser sumarizadas assim: há uma seqüência de tentativas; cada tentativa apresenta um estímulo como modelo e dois ou mais estímulos de comparação; para cada modelo, há somente um estímulo de comparação correto; os modelos variam entre as tentativas e, portanto, o estímulo de comparação correto também muda ao longo das tentativas; a seqüência de tentativas é imprevisível para o participante, o que não permite estratégias como a memorização da alternativa correta; conseqüências diferenciais são programadas para respostas corretas e incorretas. Os estímulos utilizados são agrupados em conjuntos (comumente designados por letras maiúsculas tais como A, B, $\mathrm{C}$, etc.) contendo dois ou mais membros (designados individualmente por combinações alfanuméricas). Assim, por exemplo, um conjunto A agrupa os estímulos A1, A2, ..., An; um conjunto $\mathrm{B}$ agrupa os estímulos $\mathrm{B} 1, \mathrm{~B} 2, \ldots$, $\mathrm{Bn}$; um conjunto $\mathrm{C}$ agrupa os estímulos $\mathrm{C} 1, \mathrm{C} 2, \ldots, \mathrm{Cn}$. Por convenção, denomina-se $\mathrm{AB}$ a relação entre os estímulos modelo do conjunto A e os estímulos de comparação do conjunto B: na presença do modelo A1, a escolha do estímulo de comparação B1 é indicada como correta, na presença do modelo A2, a escolha do estímulo de comparação B2 é indicada como correta, e assim por diante. Quando são ensinadas, por exemplo, as relações $\mathrm{AB}$ e
$\mathrm{BC}$, com três membros em cada um dos conjuntos $\mathrm{A}, \mathrm{B}$ e C, participantes humanos geralmente mostram relações emergentes que atestam a formação de três classes de estímulos equivalentes envolvendo os membros relacionados de cada conjunto (A1, B1 e C1 formam uma classe, A2, B2 e C2 formam outra classe e A3, B3 e C3 formam uma terceira classe).

As classes de estímulos equivalentes geradas pelo treino de relações entre estímulos, como em $\mathrm{AB}$ e $\mathrm{BC}$, são demonstradas por relações emergentes que atestam as propriedades de reflexividade, de simetria e de transitividade das relações ensinadas. A reflexividade é demonstrada pelo estabelecimento de uma relação entre um determinado estímulo e ele mesmo. Por exemplo, tendo A 1 como modelo e A1, A2 e A3 como estímulos de comparação, o participante deve selecionar A1. Testes de simetria podem ser feitos pela inversão da ordem de uma relação treinada. Por exemplo, tendo B1 como modelo e A1, A2 e A3 como estímulos de comparação, o participante deve selecionar A1. A transitividade é determinada pela demonstração de que dois pares de estímulos com um elemento comum estabelecem um novo par. Por exemplo, tendo A1 como modelo e C1, C2 e C3 como estímulos de comparação, o participante deve selecionar C1. Simetria e transitividade podem ser testadas conjuntamente. Tendo, por exemplo, $\mathrm{C} 1$ como modelo e como comparações A1, A2 e A3, a seleção do participante deve ser A1. Testes que combinam simetria e transitividade são normalmente chamados de testes de equivalência porque demonstram, simultaneamente, duas propriedades das relações de equivalência. A terceira propriedade (reflexiva) é normalmente assumida nos estudos com participantes humanos (cf. Saunders \& Green, 1999).

Utilizando variações dessa metodologia, um grande número de trabalhos experimentais tem apontado e fortalecido congruências entre relações de equivalência e propriedades definidoras da linguagem simbólica (e.g., J. C. de Rose, McIlvane, Dube, Galpin, \& Stoddard, 1988; Dougher, Augustson, Markham, Greenway, \& Wulfert, 1994; Hayes, Kohlenberg, \& Hayes, 1991; Saunders, Saunders, Kirby, \& Spradlin, 1988; Wulfert \& Hayes, 1988). Os estímulos utilizados em estudos sobre equivalência são mais comumente visuais (geralmente figuras abstratas) ou auditivos (mais comumente sílabas ou palavras sem sentido).

Trabalhos recentes têm avaliado a especificação operacional de Sidman e colaboradores por meio de procedimentos externos ao modelo de equivalência. Por exemplo, Barnes-Holmes et al. (2005) testaram a adequação da equivalência de estímulos na simulação de relações simbólicas por meio de um procedimento capaz de detectar um efeito conhecido como "dica semântica" (semantic priming effect). Pesquisadores da ciência cognitiva (e.g., Meyer \& Schvaneveldt, 1971; Neely, 1991) têm descrito que significados relacionados podem gerar o efeito de dica semântica sobre o desempenho de participantes em 
algumas tarefas de reconhecimento de palavras (e.g., tentativas de decisão léxica). Por exemplo, se duas palavras são apresentadas em seqüência - a primeira como "dica" (prime) e a segunda como "alvo" (target) - e se elas estão semanticamente relacionadas (e.g., tigreleão), os participantes geralmente reconhecem a palavra alvo em menos tempo do que a reconheceriam se não houvesse relação semântica entre elas (e.g., tigre-casa). Barnes-Holmes et al. (2005) verificaram que relações de equivalência podem gerar o efeito de dica semântica entre palavras sem sentido construídas arbitrariamente. Em três experimentos, os participantes responderam mais rapidamente em tentativas de decisão léxica quando o alvo era equivalente à dica do que quando dica e alvo não eram equivalentes ou quando pelo menos um dos estímulos não havia sido apresentado durante o treino. Esses resultados mostram que palavras sem sentido, quando equivalentes, podem adquirir propriedades de palavras com significados relacionados, contribuindo para dar mais validade ao modelo de equivalência de estímulos.

A validação externa do modelo de equivalência também tem sido promovida com o uso de um instrumento de diferencial semântico (e.g., Bortoloti \& de Rose, 2007; Bortoloti \& de Rose, 2009; de Rose \& Bortoloti, 2007). O diferencial semântico (Osgood, Suci, \& Tannenbaum, 1957) é uma técnica usada para medir o significado que os participantes atribuem a "conceitos" (palavras, locuções, fotografias, desenhos, etc.). O instrumento utilizado é constituído por um conjunto de es-ca-las bipolares ancoradas por adjetivos opostos. Para cada conceito apresentado, o participante deve assinalar em cada escala o quanto o conceito se aproxima de um dos adjetivos polares. Essas informações são reunidas, quantificadas e apresentadas na forma de valores numéricos que expressam sumariamente os significados dos conceitos avaliados. De posse desses valores, é possível comparar o significado de um ou de vários conceitos, para um ou vários indivíduos, em uma ou em várias situações. Em um estudo que combinava o modelo de equivalência de estímulos com o diferencial semântico, Bortoloti e de Rose (2007) demonstraram que uma figura abstrata avaliada inicialmente como neutra, quando tornada equivalente a um estímulo significativo, passava ser avaliada de modo similar a esse estímulo. A comprovação, pelo diferencial semântico, dessa mudança de significado, confere maior validade à equivalência de estímulos como modelo do significado.

Além de dar mais validade ao modelo de equivalência, o diferencial semântico tem permitido identificar parâmetros experimentais que podem interferir no nível de transferência de significados por meio de relações simbólicas simuladas em laboratório. Por exemplo, Bortoloti e de Rose (2009) apresentam dados indicando que o nível de transferência de significados é mais intenso quando as tentativas de emparelhamento ao modelo envolvem um "atraso" entre a apresentação do estímulo modelo e dos estímulos de comparação (procedimento muitas vezes chamado de delayed matching-to-sample, ou MTS atrasado). Em outro experimento descrito no mesmo trabalho, os autores mediram o efeito da "distância nodal" sobre a indução de significado entre estímulos equivalentes. Distância nodal se refere à quantidade de estímulos mediadores presentes em uma classe de equivalência. Se três conjuntos de estímulos são representados pelas letras A, B e C, a emergência da relação CA após o treino das relações $\mathrm{AB}$ e $\mathrm{BC}$ fornece uma evidência de que os estímulos relacionados dos conjuntos $\mathrm{A}, \mathrm{B}$ e $\mathrm{C}$ são equivalentes. Nesse caso, a ligação entre $\mathrm{A}$ e $\mathrm{C}$ é intermediada pelos estímulos do conjunto B. Os estímulos que estabelecem a ligação entre aqueles que não foram relacionados diretamente são chamados de nódulos. Quando se considera um treino de relações $\mathrm{AB}, \mathrm{BC}$ e $\mathrm{CD}$, dois nódulos ( $\mathrm{B}$ e $\mathrm{C})$ estabelecem a ligação entre $\mathrm{A}$ e $\mathrm{D}$. A distância nodal entre $\mathrm{A}$ e $\mathrm{C}$ é, portanto, menor do que a distância nodal entre A e D. Bortoloti e de Rose (2009) ensinaram dois grupos experimentais a estabelecer três classes de equivalência com sete membros cada. Os autores ensinaram aos participantes as relações $\mathrm{AB}, \mathrm{AC}, \mathrm{CD}$, $\mathrm{DE}, \mathrm{EF}$ e FG e testaram a emergência das relações derivadas GB e BG. Os estímulos do conjunto A eram fotografias de faces humanas expressando emoções e os estímulos dos demais conjuntos B, C, D, E, F e G eram figuras abstratas. Com esse arranjo, os estímulos $\mathrm{D}$, a um nódulo das faces, tiveram no diferencial semântico uma avaliação bem mais próxima das faces do que os estímulos F, a três nódulos de distância das faces. Variações na transferência de significados fortalecem a hipótese de que podem existir "graus de relacionamento" diferentes entre estímulos equivalentes (cf. Fields, Adams, Verhave, \& Newman, 1993).

O presente estudo pretendeu verificar a ocorrência do efeito de dica semântica em "tentativas de decisão léxica" que não envolviam palavras, mas somente estímulos abstratos equivalentes e não equivalentes. Outro objetivo foi avaliar se a magnitude do efeito de dica poderia variar em função da estrutura de treino utilizada para o estabelecimento das relações entre estímulos e comparar esses dados com os resultados da aplicação de um instrumento de diferencial semântico. Para que o diferencial semântico pudesse ser aplicado de maneira eficiente, foi programado o estabelecimento de relações de equivalência envolvendo estímulos significativos e figuras abstratas. Os estímulos significativos eram fotografias de faces expressando diferentes emoções. Neste trabalho, foram utilizadas as estruturas de treino conhecidas como "um-paramuitos" e "muitos-para-um" na geração de três classes de estímulos equivalentes envolvendo cinco conjuntos de estímulos: A, B, C, D e E. Para um grupo de participantes, denominado de Grupo Um para Muitos (GUM), as relações diretamente treinadas obedeciam à estrutura $\mathrm{AB}, \mathrm{BC}, \mathrm{CD}$ e $\mathrm{CE}$; o outro grupo experimental, denominado de Grupo Muitos para Um (GMU), foi treinado 
na estrutura DC, EC, CB e BA. Para os dois grupos, os estímulos do conjunto $\mathrm{B}$ eram fotografias de faces expressando emoções e os conjuntos A, C, D e E eram fi- guras abstratas. A Figura 1 mostra um esquema geral das relações condicionais que foram treinadas diretamente para os dois grupos de participantes.

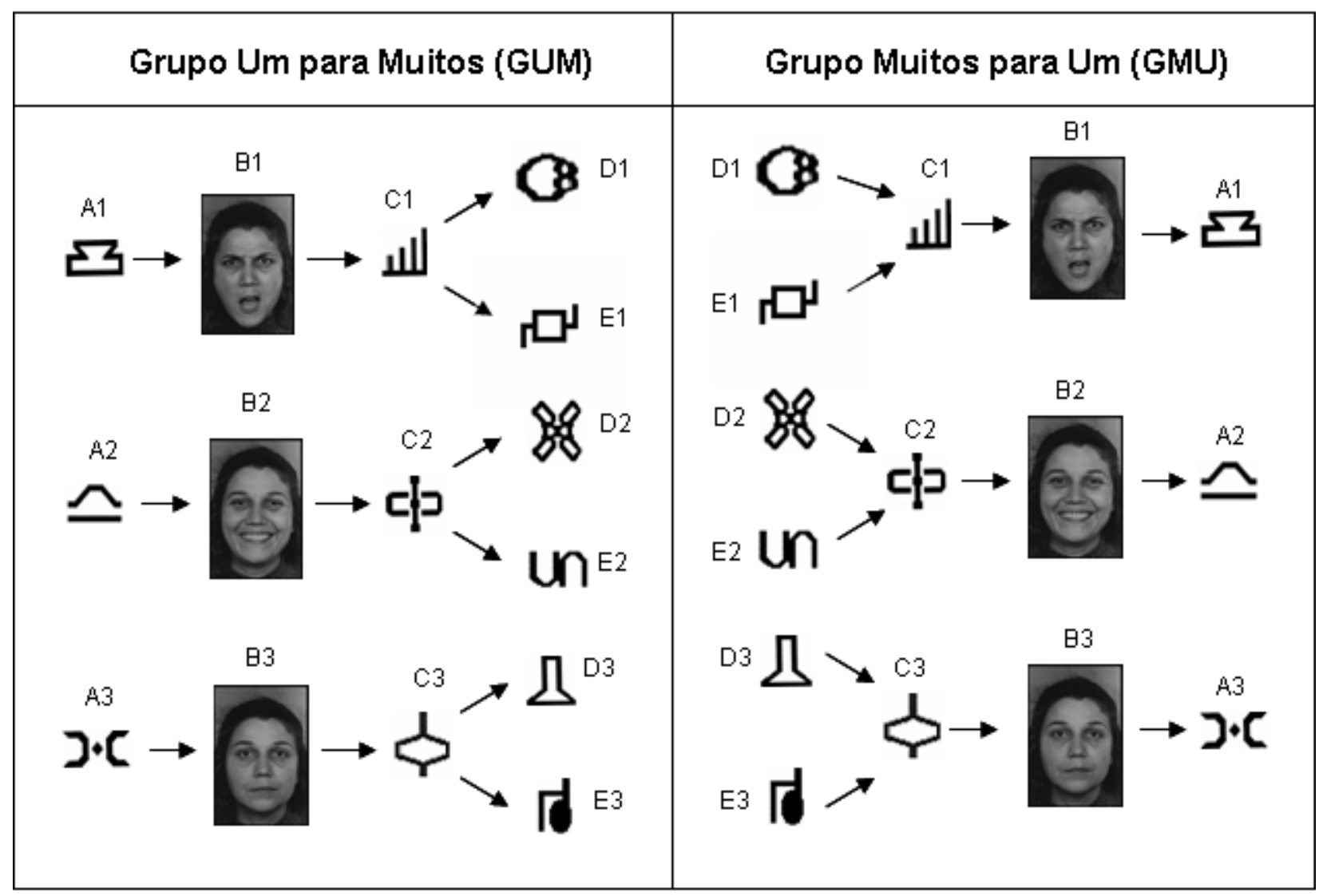

Figura 1. Diagrama esquemático da estrutura dos procedimentos de ensino para cada grupo experimental

Note-se que os estímulos envolvidos nas três classes de equivalência eram os mesmos para os dois grupos de participantes, mas que a direção das relações treinadas era diferente. Note-se, ainda, que os participantes do GUM viam os estímulos do conjunto $\mathrm{C}$ como modelos para dois conjuntos de estímulos (D e E) e que os participantes do GMU viam os estímulos do conjunto $\mathrm{C}$ como opções de escolha (ou estímulos de comparação) para dois conjuntos de estímulos modelo (D e E). Com relação à formação de equivalência de estímulos, qualquer um dos arranjos da Figura 1 pode dar origem a classes de cinco membros, envolvendo A, B, C, D e E. Teriam eles influências distintas sobre o desempenho dos participantes ante tentativas de decisão léxica e diferencial semântico?

\section{Método}

\section{Participantes}

Foram recrutados pessoalmente pelo pesquisador e divididos aleatoriamente entre dois grupos experimentais 22 estudantes recém-admitidos no curso de Graduação em Psicologia da Universidade Federal de São Carlos (UFSCar). Cada participante recebia um vale-lanche para consumir em uma lanchonete da universidade. Este estudo contou ainda com a participação de um grupo controle composto por outros 24 alunos de graduação, também recém-admitidos no curso de Psicologia da UFSCar. Os participantes do grupo controle não recebiam o vale-lanche.

\section{Situação e Equipamento}

As sessões experimentais foram conduzidas em uma sala do Departamento de Psicologia da UFSCar. A sala tem razoável isolamento acústico e de outras interferências externas. Os participantes dos dois grupos experimentais faziam atividades no computador e avaliavam figuras com um instrumento de diferencial semântico impresso em folhas de papel. Um computador Apple Macintosh G4 programado com o software MTS (Dube \& Hiris, 1997) era utilizado para treinar relações entre estímulos e testar a formação de classes de equivalência; um computador PC HP Pavilion programado com o software Muzamba 1.0 (de Drausio Capobianco, 
drausiocap@gmail.com) era utilizado nas tentativas de decisão léxica. Durante o treino e teste de relações de equivalência, os estímulos eram apresentados em até cinco "janelas" de 5 X $5 \mathrm{~cm}$ e os participantes deviam escolhê-los clicando na "janela" correspondente.

\section{Estímulos}

Fotografias de faces humanas e figuras abstratas formavam cinco conjuntos de estímulos, cada qual designado por uma das cinco primeiras letras do alfabeto (A, B, C, D ou E). O conjunto B era composto por 12 fotografias: quatro faces raivosas (B1), quatro faces alegres (B2) e quatro faces neutras (B3). Sendo B1 a designação genérica das faces raivosas, $\mathrm{B} 2$ a designação das faces alegres e B3 a designação das faces neutras, cada tentativa que apresentava o estímulo B1 podia exibir uma de quatro faces raivosas. $\mathrm{O}$ mesmo era válido para as tentativas que apresentavam B2 e B3: era exibida uma face de cada vez e elas se alternavam ao longo das tentativas. Os conjuntos A, C, D e E eram compostos por três figuras abstratas cada (conjunto A: figuras A1, A2 e A3; conjunto $\mathrm{C}$ : figuras $\mathrm{C} 1, \mathrm{C} 2 \mathrm{e} \mathrm{C} 3$; conjunto $\mathrm{D}$ : figuras $\mathrm{D} 1, \mathrm{D} 2$ e D3; conjunto E: figuras E1, E2 e E3).

\section{Procedimentos}

Fase 1: Treino de Discriminações Condicionais. O procedimento adotado para treinar relações entre estímulos foi o emparelhamento arbitrário ao modelo (MTS, de matching-to-sample). Cada tentativa de MTS era iniciada com a exposição do estímulo modelo na janela central. Um clique nessa janela fazia o programa apresentar três estímulos de comparação em três das janelas periféricas, ficando modelo e comparações presentes na mesma tela (MTS simultâneo). Um clique na janela contendo o estímulo considerado correto em função do modelo apresentado produzia o aparecimento de uma seqüência de tons e um arranjo de estrelas movendo-se na tela do computador. Respostas incorretas produziam o escurecimento da tela por três segundos. Seguia-se um intervalo entre tentativas de dois segundos, depois do qual a tentativa seguinte tinha início.

Os dois grupos experimentais foram ensinados a estabelecer discriminações condicionais para gerar classes de equivalência entre fotografias de faces expressando diferentes emoções e conjuntos de figuras abstratas. A Figura 2 ilustra uma tentativa de treino de discriminação condicional.

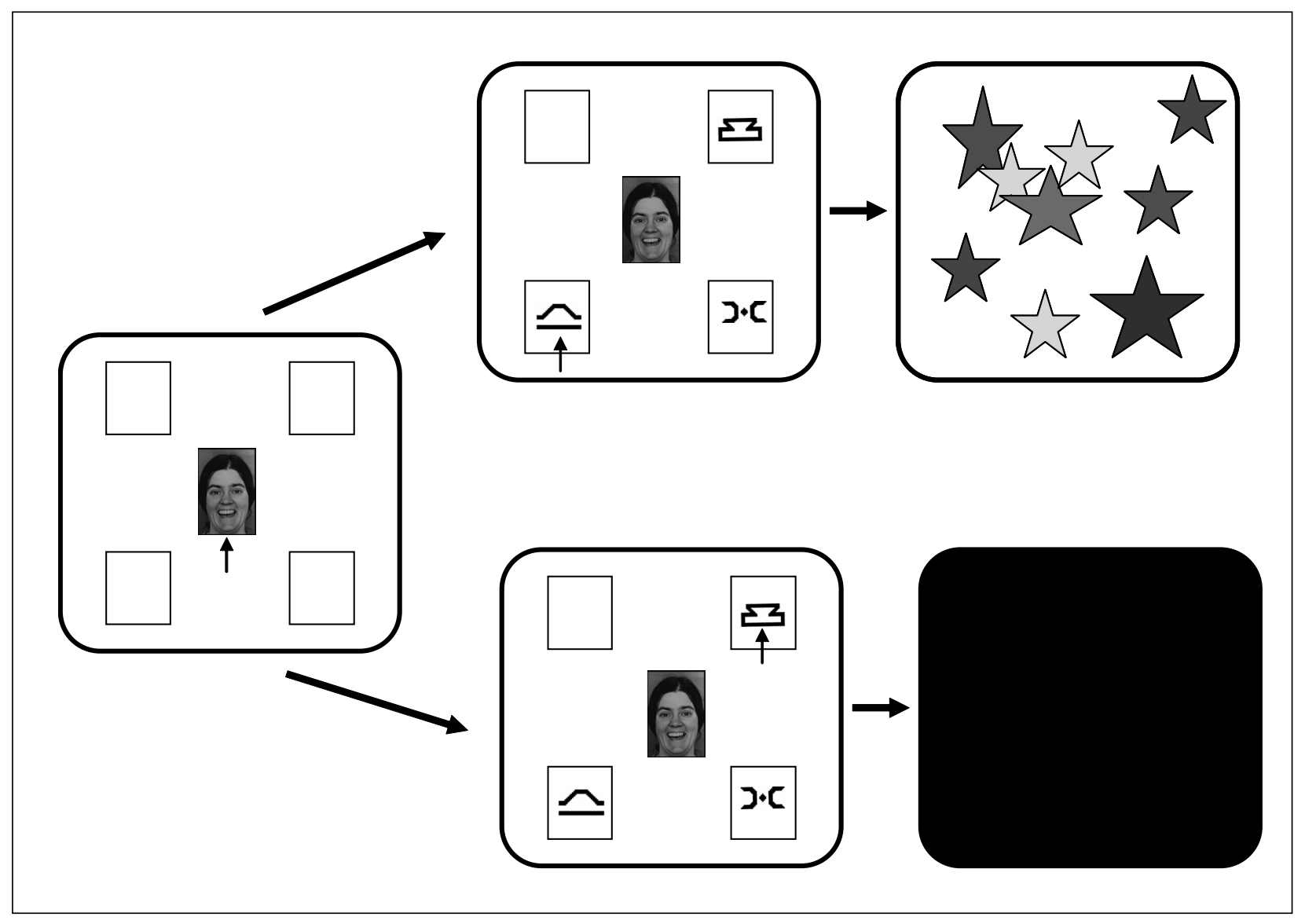

Figura 2. Exemplo de tentativa de treino de discriminação condicional

Nota. Primeiro, um estímulo era apresentado no centro da tela e o participante devia clicar nele. Em seguida, dois outros estímulos eram apresentados como opções de escolha. A escolha correta (parte superior da figura) era consequenciada com estrelas e sons; escolhas incorretas (parte inferior da figura) eram consequenciadas com uma tela escura. 
As relações eram apresentadas seqüencialmente, em blocos de tentativas que obedeciam a estruturas de treino distintas. Um grupo de participantes, denominado de Grupo Um para Muitos (GUM), foi treinado a estabelecer relações condicionais numa estrutura $\mathrm{AB}, \mathrm{BC}, \mathrm{CD}$ e CE; o outro grupo experimental, denominado de Grupo Muitos para Um (GMU), foi treinado na estrutura DC, EC, CB e BA (ver Figura 1).

Para o GUM, a discriminação condicional AB foi ensinada em primeiro lugar; para o GMU, a discriminação condicional DC foi ensinada primeiro. Cada discriminação condicional era treinada inicialmente em um bloco de 36 tentativas. Por exemplo, para o GMU, cada tentativa DC podia apresentar D1, D2 ou D3 como modelo e apresentava $\mathrm{C} 1, \mathrm{C} 2$ e C3 como opções de escolha. Os modelos eram exibidos na janela central numa seqüência randomizada, com a restrição de que cada um deles aparecia em um total de 12 tentativas e não ocorria em mais de duas tentativas consecutivas. Os estímulos de comparação $\mathrm{C} 1, \mathrm{C} 2$ e C3 eram apresentados em três janelas periféricas, ficando a outra janela periférica sem nenhuma figura. A posição de cada figura e da janela vazia (sem figura) mudava de tentativa para tentativa, de acordo com uma seqüência randomizada. Particularmente, a posição da janela que continha a figura designada como correta mudava a cada tentativa, de acordo com uma seqüência randomizada. As primeiras doze tentativas do bloco apresentavam, além dos estímulos modelo e de comparação, uma instrução escrita, acima do modelo, onde se lia: "Quando esta figura estiver aqui" e acima do estímulo de comparação correto aparecia escrito "escolha esta". Essas instruções deixavam de ser apresentadas a partir da $13^{\text {a }}$ tentativa do bloco. Esse bloco de 36 tentativas envolvia, portanto, uma seqüência de 12 tentativas com instrução escrita e 24 tentativas sem instrução. O bloco era repetido até que o participante fizesse escolhas corretas em todas as tentativas. Quando esse critério era atingido, considerava-se encerrado o ensino da relação DC e passava-se ao ensino da relação EC, procedido de maneira análoga. Atingido o critério de escolhas corretas em todas as tentativas do tipo EC, passava-se ao ensino da relação CB e, em seguida, ao ensino da relação BA com procedimento semelhante. O mesmo procedimento era aplicado aos participantes do GUM no treinamento inicial das relações $\mathrm{AB}, \mathrm{BC}, \mathrm{CD}$ e $\mathrm{CE}$. A diferença mais relevante entre os tratamentos dispensados aos dois grupos consistia, portanto, na estrutura de treino de algumas relações: para o GUM, um conjunto de estímulos modelo (C) era relacionado a dois conjuntos de estímulos de comparação (D e E); para o GMU, dois conjuntos de modelos (D e E) eram relacionados a um conjunto de comparações $(C)$.

Os participantes eram submetidos, em seguida, a um bloco de 48 tentativas que reunia de maneira intercalada e randômica os quatro conjuntos de relações ensinadas, $\mathrm{AB}, \mathrm{BC}, \mathrm{CD}$ e CE (para o GUM) ou DC, EC, CB e BA (para o GMU). Esse bloco era repetido até que o participante não apresentasse mais do que dois erros. Atingido o critério, aparecia escrito na tela "O computador não vai mais sinalizar se as suas escolhas estão corretas ou erradas" e o bloco era repetido sem o feedback que consequenciava as escolhas do participante. Esse bloco também era repetido até que não mais do que dois erros fossem apresentados. Se o participante errasse em mais do que cinco tentativas, ele era submetido novamente ao bloco anterior (com feedback) antes de repetir o atual. Atingido o critério no bloco sem feedback, o participante passava à fase seguinte, realizada no outro computador.

Fase 2: Tentativas de Decisão Léxica. Os tempos de apresentação de cada estímulo e a estrutura geral das tentativas de decisão léxica foram programados com base na descrição de Barnes-Holmes et al. (2005). A cor de fundo da tela do computador permanecia branca durante todo o procedimento. Cada tentativa começava com um estímulo de advertência, o desenho de uma mão espalmada vermelha no centro da tela. Esse estímulo de advertência permanecia na tela por $500 \mathrm{~ms}$ e era, então, substituído pela dica (e.g., a figura abstrata A1), que permanecia na tela por $200 \mathrm{~ms}$. Quando a dica era removida, havia um intervalo de $50 \mathrm{~ms}$ durante o qual a tela permanecia vazia e, então, o estímulo alvo (e.g., a figura abstrata C1) era apresentado. Depois de $1500 \mathrm{~ms}$, o alvo era removido e o desenho de uma mão espalmada verde era apresentada no centro da tela. Finalmente, depois de $1250 \mathrm{~ms}$, a mão verde era substituída pela mão vermelha e a próxima tentativa tinha início. Cada participante era submetido a um total de 108 tentativas estruturadas dessa forma.

Os participantes respondiam usando o teclado, devendo deixar seus dedos indicadores direito e esquerdo posicionados sobre, respectivamente, as teclas " $\mathrm{M}$ " e "C". Eles eram instruídos a pressionar a tecla M (para sim) se tivessem visto a segunda figura (o estímulo alvo) na fase anterior e a pressionar a tecla $\mathrm{C}$ (para não) caso não tivessem visto a segunda figura em momento algum do experimento. A localização (à direita ou à esquerda) das teclas "sim" e "não" era balanceada entre os participantes e as instruções eram adaptadas apropriadamente. Era enfatizado que as respostas deveriam ser dirigidas somente ao alvo, não à dica e era pedido que o participante respondesse da maneira mais rápida e acurada possível em cada tentativa. Foram consideradas válidas as respostas que ocorriam dentro do intervalo compreendido entre a apresentação do estímulo alvo e a remoção da mão verde. Respostas ocorridas fora desse período eram desconsideradas nas análises subsequentes.

As instruções eram dadas verbalmente pelo experimentador, durante uma breve sessão de treino composta por 12 tentativas que não foram consideradas na análise de dados. Durante as tentativas de decisão léxica consideradas, os participantes eram expostos a 54 pares de estímulos: 30 pares da mesma relação de equivalência e 24 pares que eram de relações diferentes ou que continham uma ou duas figuras novas. A Tabela 1 contém uma representação esquemática de todos os 54 tipos de tentativas divididos em nove categorias. 
Bortoloti, R. \& Rose, J. C. C. (2011). Avaliação do Efeito de Dica Semântica e da Indução de Significado entre Estímulos Abstratos Equivalentes.

Tabela 1

Esquema Geral dos 54 Tipos de Tentativas de Decisão Léxica

\begin{tabular}{|c|c|c|c|c|c|c|c|c|c|c|c|c|c|c|}
\hline \multicolumn{3}{|c|}{ Classe-classe } & \multicolumn{3}{|c|}{$\begin{array}{l}\text { Classe-não } \\
\text { classe }\end{array}$} & \multicolumn{3}{|c|}{$\begin{array}{l}\text { Classe-estímulo } \\
\text { novo }\end{array}$} & \multicolumn{3}{|c|}{$\begin{array}{r}\text { Estímulo } \\
\text { novo-classe }\end{array}$} & \multicolumn{3}{|c|}{$\begin{array}{l}\text { Estímulo } \\
\text { novo-estímulo }\end{array}$} \\
\hline S1 & $\mathrm{S} 2$ & $\mathrm{R}$ & $\mathrm{S} 1$ & $\mathrm{~S} 2$ & $\mathrm{R}$ & $\mathrm{S} 1$ & $\mathrm{~S} 2$ & $\mathrm{R}$ & $\mathrm{S} 1$ & $\mathrm{~S} 2$ & $\mathrm{R}$ & $\mathrm{S} 1$ & S2 & $\mathrm{R}$ \\
\hline \multicolumn{15}{|c|}{$\begin{array}{l}\text { Treino (GUM) } \\
\text { ou Simetria (GMU) }\end{array}$} \\
\hline $\mathrm{C} 1$ & D1 & $\mathrm{S}$ & $\mathrm{D} 2$ & $\mathrm{C} 1$ & $\mathrm{~S}$ & $\mathrm{D} 2$ & N1 & $\mathrm{N}$ & N1 & D2 & $\mathrm{S}$ & N1 & N6 & $\mathrm{N}$ \\
\hline $\mathrm{C} 1$ & E1 & $\mathrm{S}$ & D3 & $\mathrm{C} 2$ & $\mathrm{~S}$ & $\mathrm{C} 3$ & $\mathrm{~N} 2$ & $\mathrm{~N}$ & $\mathrm{~N} 2$ & $\mathrm{C} 3$ & $\mathrm{~S}$ & $\mathrm{~N} 2$ & N5 & $\mathrm{N}$ \\
\hline $\mathrm{C} 2$ & D2 & $\mathrm{S}$ & $\mathrm{A} 1$ & D3 & $\mathrm{S}$ & $\mathrm{A} 1$ & N3 & $\mathrm{N}$ & $\mathrm{N} 3$ & A1 & $\mathrm{S}$ & N3 & N4 & $\mathrm{N}$ \\
\hline $\mathrm{C} 2$ & E2 & $\mathrm{S}$ & $\mathrm{A} 2$ & D1 & $\mathrm{S}$ & E1 & $\mathrm{N} 4$ & $\mathrm{~N}$ & N4 & E1 & $\mathrm{S}$ & N4 & N3 & $\mathrm{N}$ \\
\hline $\mathrm{C} 3$ & D3 & $\mathrm{S}$ & E1 & A2 & $\mathrm{S}$ & $\mathrm{C} 3$ & N5 & $\mathrm{N}$ & N5 & $\mathrm{C} 3$ & $\mathrm{~S}$ & N5 & $\mathrm{N} 2$ & $\mathrm{~N}$ \\
\hline C3 & E3 & $\mathrm{S}$ & E3 & A1 & $\mathrm{S}$ & $\mathrm{A} 2$ & N6 & $\mathrm{N}$ & N6 & A2 & $\mathrm{S}$ & N6 & N1 & $\mathrm{N}$ \\
\hline
\end{tabular}

Simetria (GUM)

ou Treino (GMU)

D1 $\quad$ C1 $\quad \mathrm{S}$

E1 C1 S

D2 $\mathrm{C} 2 \quad \mathrm{~S}$

E2 $\mathrm{C} 2 \quad \mathrm{~S}$

D3 $\mathrm{C} 3 \quad \mathrm{~S}$

E3 $\quad$ C3 $\quad \mathrm{S}$

Transitividade (GUM)

ou Equivalência I (GMU)

A1 $\quad$ C1 $\quad$ S

A1 E1 S

A2 $\mathrm{C} 2 \quad \mathrm{~S}$

A2 $22 \quad S$

A3 C3 S

A3 E3 $S$

Equivalência I (GUM)

ou Transitividade (GMU)

C1 A1 S

E1 A1 S

C2 A2 S

E2 A2 S

C3 A3 S

E3 A3 S

Equivalência II(GUM e GMU)

D1 E1 S

D2 E2 S

D3 E3 S

E1 D1 S

E2 D2 S

E3 D3 S

Nota. $\mathrm{S} 1$ = dica, $\mathrm{S} 2$ = alvo, $\mathrm{R}=$ resposta correta, $\mathrm{Nn}=$ estímulo novo. 
Os 54 tipos de tentativas eram apresentados em ordem randômica por duas vezes. Depois das 108 tentativas de decisão léxica, o participante voltava ao primeiro computador para os testes de equivalência.

Fase 3: Testes de Equivalência. Nesta etapa, para verificar a manutenção da linha de base depois das tentativas de decisão léxica, os participantes eram submetidos novamente ao bloco de 48 tentativas sem feedback que reunia os quatro conjuntos de relações ensinadas, $\mathrm{AB}, \mathrm{BC}, \mathrm{CD}$ e CE (para o GUM) ou DC, EC, CB e BA (para o GMU). Em seguida, outro bloco de 48 tentativas de sonda de equivalência sem feedback testava o estabelecimento das relações derivadas EA, DA, DE e ED (para o GUM) ou AE, AD, DE e ED (para o GMU). O participante deveria acertar pelo menos 44 das 48 tentativas de sonda de equivalência para avançar à próxima fase.

Fase 4-Avaliação de Estímulos com Escalas Bipolares. Nesta etapa, os participantes com desempenhos consistentes no bloco de sonda dos dois grupos experimentais deviam avaliar os estímulos abstratos dos conjuntos $\mathrm{D}$ e E de acordo com uma série de escalas bipolares. Cada escala se compunha de sete intervalos e era ancorada em suas duas extremidades por "termos polares" constituindo um par de adjetivos antônimos. Vistas em conjunto, representavam uma série de contínuos que iam de um adjetivo ao seu oposto. Cada conjunto trazia acima de si a reprodução de um estímulo e o participante deveria assinalar o lugar dele em cada escala, conforme é exemplificado na Figura 3.

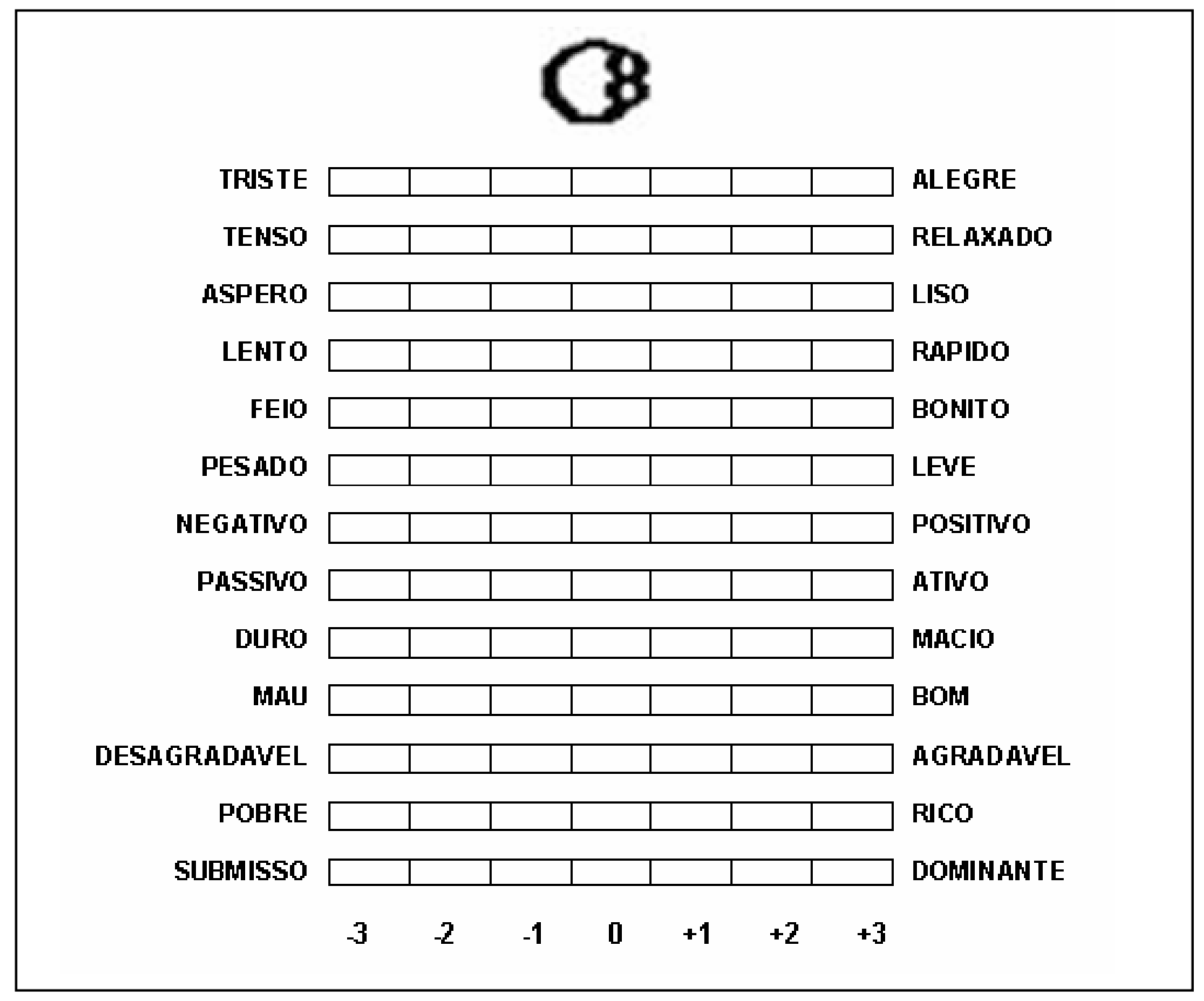

Figura 3. Exemplo de estímulo abstrato e escalas utilizadas pelos participantes nas avaliações dele

Quanto mais forte a relação que o participante atribuísse à figura impressa no alto da folha e um adjetivo, mais próximo dele deveria ser assinalado o espaço na escala correspondente. Se o participante achasse que a figura não tinha qualquer relação com os adjetivos de um determinado par, deveria assinalar o espaço central nessa escala, ao qual foi atribuído, para propósito de análise de dados, o valor 0 (zero). Aos outros intervalos foram 
atribuídos valores que variavam de -3 a +3 , sendo -3 correspondente à posição mais próxima ao adjetivo negativo $\mathrm{e}+3$ correspondente à posição mais próxima do adjetivo positivo. Para explicitar esses valores, a Figura 4 foi montada com os adjetivos considerados negativos sempre à esquerda e os positivos sempre à direita. $\mathrm{Na}$ folha entregue aos participantes, essa disposição era randomizada e os valores que aparecem abaixo das escalas não estavam presentes. As instruções para o preenchimento das escalas podem ser solicitadas aos autores.

\section{Grupo Controle}

Os participantes do grupo controle deviam avaliar as fotografias de expressões faciais usando o mesmo conjunto de escalas bipolares utilizado pelos participantes dos dois grupos experimentais. Não foi ensinada a esses participantes qualquer relação entre as faces e outros estímulos.

\section{Resultados}

Cinco dos 22 participantes recrutados não atingiram todos os critérios estabelecidos nos blocos de linha de base e foram dispensados de prosseguir no experimento. Entre os 17 participantes restantes, sete não passaram nos testes de equivalência (menos de 44 tentativas corretas entre as 48 apresentadas; média de tentativas corretas $=36,71$; amplitude de variação $=21$ a 43 ) realizados depois das tentativas de decisão léxica. Três deles pertenciam ao GUM e quatro ao GMU. Os desempenhos dos participantes que passaram nos testes de equivalência foram bastante similares durante as tentativas de decisão léxica. A Tabela 2 apresenta uma análise descritiva dos tempos médios para a emissão de respostas corretas nas tentativas de decisão léxica entre aqueles que passaram (sonda consistente) e entre os que não passaram (sonda inconsistente) nos testes de equivalência.

Tabela 2

Os Nove Tipos de Tentativas e Estatísticas Descritivas Relacionadas a eles para os Participantes que Passaram (Sonda Consistente) e para os Participantes que Falharam (Sonda Inconsistente) nos Testes de Equivalencia

\begin{tabular}{|c|c|c|c|c|c|c|c|c|}
\hline \multirow[b]{3}{*}{ Estímulos apresentados } & \multirow{4}{*}{ 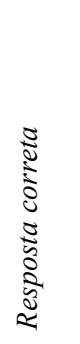 } & \multirow{4}{*}{ 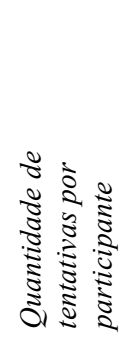 } & \multicolumn{4}{|c|}{ Tempo de reação (ms) } & \multirow{2}{*}{\multicolumn{2}{|c|}{ Porcentagem de erros }} \\
\hline & & & \multicolumn{2}{|c|}{ Média } & \multicolumn{2}{|c|}{ Desvio padrão } & & \\
\hline & & & こั & 气ั & ฐัฐ & 气ั̆ & \multirow{2}{*}{ 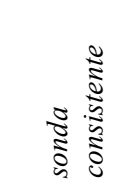 } & \multirow{2}{*}{ 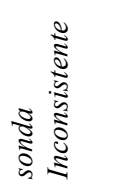 } \\
\hline Relação dica-alvo & & & $\begin{array}{ll}0 \\
0 \\
0 \\
0\end{array}$ & $\begin{array}{ll}\widetilde{0} \\
\overline{0} \\
0 \\
\vdots\end{array}$ & $\begin{array}{ll}0 \\
0 \\
0\end{array}$ & $\begin{array}{l}\bar{\delta} \\
\bar{\Xi} \\
\vdots\end{array}$ & & \\
\hline \multicolumn{9}{|l|}{ Pares equivalentes } \\
\hline 1. Treino direto & Sim & 12 & 698,29 & 682,62 & 172,50 & 150,98 & 0,8 & 0,0 \\
\hline 2. Simetria & Sim & 12 & 711,07 & 684,48 & 140,72 & 195,38 & 0,0 & 1,2 \\
\hline 3. Transitividade & Sim & 12 & 702,12 & 703,93 & 176,10 & 183,55 & 0,0 & 3,6 \\
\hline 4. Equivalência 1 & Sim & 12 & 720,84 & 698,15 & 202,76 & 185,73 & 3,0 & 0,0 \\
\hline 5. Equivalência 2 & Sim & 12 & 722,67 & 717,15 & 180,42 & 186,59 & 0,8 & 2,4 \\
\hline \multicolumn{9}{|l|}{ Pares não-equivalentes } \\
\hline 6. Classes diferentes & Sim & 12 & 724,84 & 701,86 & 212,12 & 207,97 & 1,7 & 0,0 \\
\hline 7. Estímulo novo como alvo & Não & 12 & 715,38 & 740,58 & 143,62 & 184,70 & 5,8 & 8,3 \\
\hline 8. Estímulo novo como dica & Sim & 12 & 794,96 & 744,30 & 185,16 & 243,09 & 5,0 & 1,2 \\
\hline 9. Dois estímulos novos & Não & 12 & 681,98 & 700,93 & 157,35 & 177,76 & 5,8 & 13,1 \\
\hline
\end{tabular}

Uma análise de variância (ANOVOA, one-way) indicou a existência de diferenças significativas entre os desempenhos dos participantes em tipos de tentativas distintos. Foi, então, conduzida uma série de testes de Tukey-Kramer para comparações múltiplas envolvendo os desempenhos dos participantes com sonda consistente e inconsistente nos nove tipos de tentativas que foram conduzidas neste estudo. As diferenças mais relevantes encontradas após essa série de testes comparativos estão apresentadas na Tabela 3.
Os participantes que passaram nos testes de equivalência responderam mais rapidamente aos pares dica-alvo que eram direta ou indiretamente relacionados do que aos pares que continham um estímulo novo como dica. Em contraste, os participantes que não passaram nos testes de equivalência responderam mais rapidamente apenas aos pares que foram diretamente treinados. 
Tabela 3

Diferenças Mais Relevantes Encontradas Após Testes de Tukey-Kramer para Comparações Múltiplas

Relações dica-alvo comparadas

Nivel de significância

da diferença entre as médias

sonda consistente

sonda Inconsistente

Treino direto $\mathrm{x}$ Estímulo novo como dica $(1 \times 8)$

Simetria $\mathrm{x}$ Estímulo novo como dica $(2 \times 8)$

Transitividade $\mathrm{x}$ Estímulo novo como dica $(3 \times 8)$

Equivalência 1 x Estímulo novo como dica $(4 \times 8)$

Equivalência $2 \times$ Estímulo novo como dica $(5 \times 8)$

Classes diferentes $\mathrm{x}$ Estímulo novo como dica $(6 \times 8)$

$\begin{array}{ll}p<0,001(* * *) & p<0,05(*) \\ p<0,01(* *) & p>0,05(\mathrm{~ns}) \\ p<0,01(* *) & p>0,05(\mathrm{~ns}) \\ p<0,05(*) & p>0,05 \text { (ns) } \\ p<0,05(*) & p>0,05 \text { (ns) } \\ p>0,05 \text { (ns) } & p>0,05 \text { (ns) }\end{array}$

\section{Diferencial Semântico}

A Figura 4 mostra as medianas dos valores atribuídos, em cada uma das escalas, às expressões faciais de alegria (gráficos à esquerda) e de raiva (gráficos à direita) pelos participantes do grupo controle. A figura apresenta também as medianas dos valores atribuídos às figuras abstratas dos conjuntos $\mathrm{D}$ e $\mathrm{E}$ equivalentes às faces alegres (à esquerda) e raivosas (à direita). A parte superior da Figura 4 apresenta as avaliações do Grupo Um-paraMuitos e a parte inferior apresenta as avaliações do Grupo Muitos-para-Um. Essas avaliações das figuras equivalentes foram feitas pelos participantes dos dois grupos experimentais que tiveram desempenhos consistentes nos blocos de sonda.

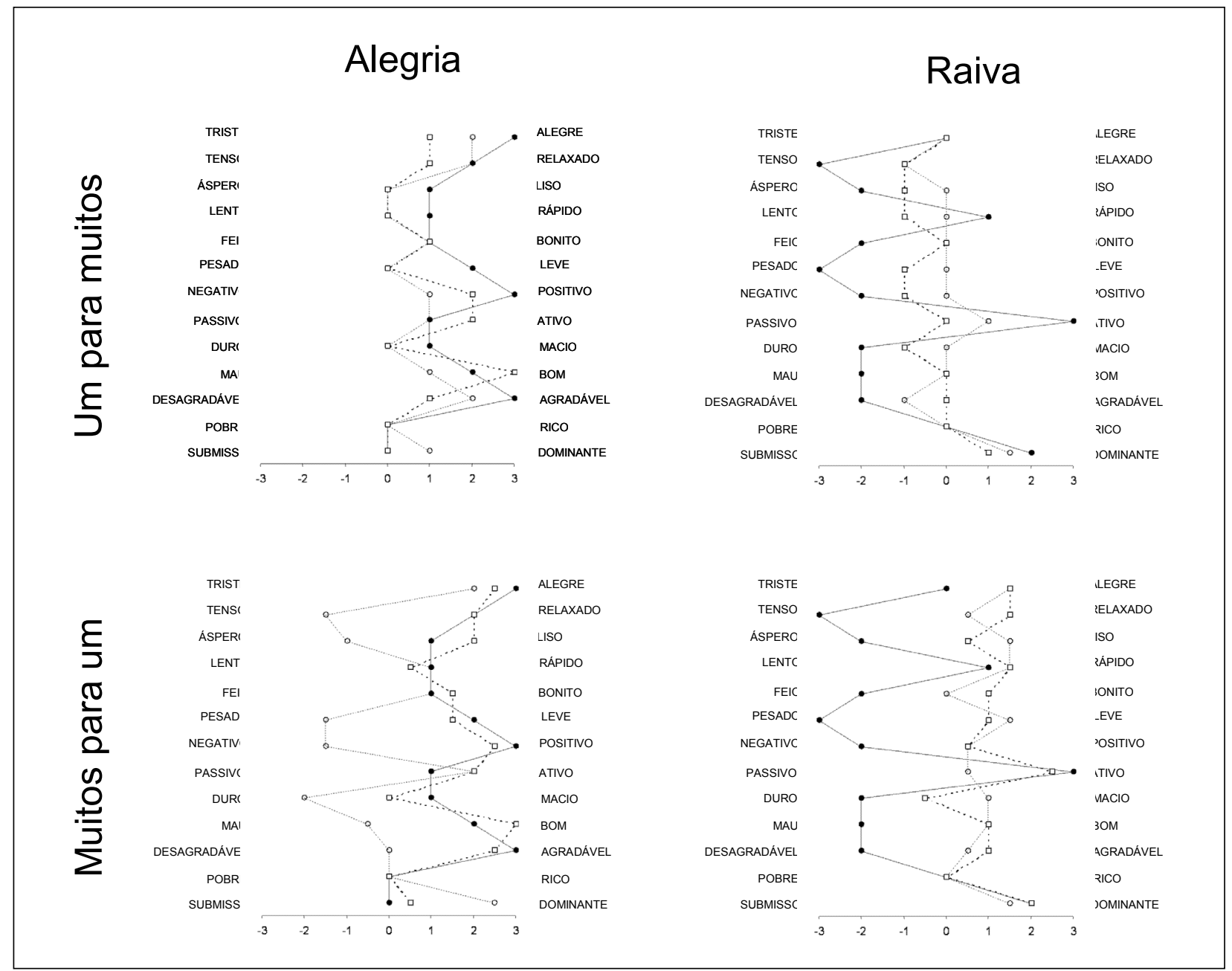

Figura 4. Medianas das avaliações das faces alegres e raivosas feitas pelos participantes do grupo controle (linhas contínuas) e dos estímulos D e E (linhas tracejadas) feitas pelos dois grupos experimentais 
Os participantes do Grupo Controle avaliaram as faces alegres de forma positiva e as faces raivosas de forma negativa. Similarmente, os participantes do GUM avaliaram as figuras equivalentes às faces alegres de forma positiva e as figuras equivalentes às faces raivosas de forma negativa. Houve muita variabilidade nas avaliações das figuras pelo GMU, que não foram similares às avaliações das faces.

\section{Discussão}

O presente estudo verificou a ocorrência do efeito de dica semântica entre estímulos relacionados utilizando somente figuras abstratas em tentativas de decisão léxica que eram conduzidas antes dos testes formais de equivalência. $\mathrm{O}$ efeito de dica foi observado entre os estímulos direta ou indiretamente relacionados para os participantes que passaram nos testes de equivalência, independentemente da estrutura de treino usada, e não foi observado entre os participantes que falharam nos testes. O efeito de dica também não foi observado quando os estímulos já tinham sido vistos anteriormente, mas pertenciam a classes diferentes. Esses resultados indicam que estímulos abstratos equivalentes podem adquirir propriedades semelhantes às de palavras com significados relacionados na linguagem natural, reforçando a equivalência de estímulos como modelo comportamental do processo semântico.

As tentativas de decisão léxica ocorreram antes dos testes de equivalência para evitar que mais estímulos abstratos de cada classe fossem emparelhados repetidamente (i.e., associados diretamente) durante os testes. Esses emparelhamentos repetidos poderiam enfraquecer eventuais interpretações que apontassem, nesta investigação, a ocorrência de um efeito de dica mediada. $\mathrm{O}$ efeito de dica mediada ocorre quando a dica e o alvo são relacionados indiretamente, via uma palavra ou um conceito mediador (e.g., Balota \& Lorch, 1986; Weisbrod et al., 1999). Por exemplo, se a palavra "listra" tem efeito de dica sobre a palavra "tigre" e se "tigre" tem efeito de dica sobre a palavra "leão", "listra" pode ter efeito de dica sobre "leão" em função do elo estabelecido pela palavra "tigre". Neste estudo, o efeito de dica obtido, por exemplo, entre os estímulos dos conjuntos A e D pode ser interpretado como dica mediada porque os estímulos A e D foram indiretamente relacionados, via estímulos B e C.

Para utilizar o diferencial semântico neste estudo, foram treinadas relações entre estímulos significativos e abstratos, simulando relações entre "referentes" e "símbolos". Expressões faciais foram escolhidas como referentes por sua natural relevância em processos atencionais (e.g., Dimberg, Thunberg, \& Elmehed, 2000; Esteves, Dimberg, \& Öhman, 1994; Morris, Öhman, \& Dolan, 1998; Öhman, 2002) e por serem estímulos que costumam receber qualificações diferentes quando submetidos ao diferencial semântico; por exemplo, em trabalhos anteriores, faces alegres receberam avaliações positivas e faces raivosas receberam avaliações negativas (e.g., Bortoloti, 2007; Bortoloti \& de Rose, 2007, 2009; de Rose \& Bortoloti, 2007). Outra característica deste estudo é a combinação de classes de equivalência com classes perceptuais (e.g., Fields, Matneja, et al., 2002; Fields, Reeve, et al., 2002). Os estímulos designados como B1, B2 e B3 não eram estímulos individuais; cada um deles envolvia quatro fotografias de faces de pessoas diferentes. O elemento comum entre as faces era a emoção expressada que em B1 era raivosa, em B2 era alegre e em B3 era neutra. Classes perceptuais foram utilizadas para garantir a equivalência de estímulos abstratos com expressões emocionais e não com aspectos idiossincráticos de faces particulares.

Conforme era esperado, as faces alegres foram avaliadas de forma positiva e as faces raivosas de forma negativa pelos participantes do Grupo Controle. De maneira semelhante, os participantes do GUM avaliaram as figuras equivalentes às faces alegres de forma positiva e as figuras equivalentes às faces raivosas de forma negativa, demonstrando que figuras abstratas podem adquirir o significado de seus referentes via relações de equivalência. Houve muita variabilidade entre as avaliações das figuras pelo GMU, que não foram similares às avaliações das faces. Esses resultados não confirmam uma hipótese inicial deste trabalho, baseada em alguns estudos que têm descrito a estrutura de treino muitos-para-um como mais eficiente na geração de classes de equivalência do que a estrutura um-para-muitos (ver, por exemplo, Saunders \& Green, 1999 para uma revisão). Esperava-se que essa suposta maior efetividade da estrutura muitos-para-um tivesse efeito sobre a transferência de significado avaliada pelo diferencial semântico e que o GMU conferisse às figuras significados mais próximos aos de seus referentes do que o GUM. O fato de essa hipótese não ter sido confirmada não parece, contudo, indicar a estrutura umpara-muitos como mais eficiente. Neste trabalho, a quantidade de erros que os participantes cometeram nas tentativas de linha de base e nas tentativas de teste de relações emergentes não variou significativamente em função da estrutura de treino empregada, sendo os dois arranjos igualmente eficientes na geração de classes de equivalência. Além disso, os desempenhos dos participantes dos dois grupos experimentais foram muito semelhantes nas tentativas de decisão léxica; a magnitude do efeito de dica não sofreu variações em função da estrutura de treino a que cada grupo foi exposto.

A justificativa para os desempenhos diferentes registrados entre os dois grupos talvez não possa ser baseada na estrutura de treino, mas na direção das relações construídas entre os referentes e os símbolos avaliados no diferencial semântico. Os participantes do GUM podiam estabelecer relações entre os significados das faces (B) e dos estímulos $\mathrm{D}$ e $\mathrm{E}$ por transitividade (as relações treinadas eram $\mathrm{AB}, \mathrm{BC}, \mathrm{CD}$ e $\mathrm{CE}$ ) enquanto os participantes do GMU deviam relacionar os significados das faces (B) 
com os estímulos $\mathrm{D}$ e $\mathrm{E}$ por transitividade $e$ simetria (as relações treinadas eram DC, EC, CB e BA). Uma evidência de que a direção das relações derivadas pode ter efeitos diferentes sobre o grau de relacionamento de estímulos equivalentes foi apresentada por Barnes-Holmes et al. (2005). Analisando a magnitude do efeito de dica semântica em função de diversas formas de relacionar estímulos, os autores encontraram diferenças significativas entre relações treinadas diretamente e relações de equivalência (transitividade + simetria), mas não entre relações treinadas diretamente e relações transitivas. Eles sugeriram que a força relativa do efeito de dica mediada pode depender da direção da relação e não simplesmente da presença de estímulos mediadores (se a direção fosse irrelevante, então os pares dica-alvo relacionados tanto por transitividade quanto por equivalência deveriam produzir tempos de reação significativamente mais lentos do que aqueles observados diante dos pares compostos por estímulos relacionados diretamente). Similarmente, no presente estudo, os participantes do GMU podem ter estabelecido uma relação menos intensa entre o significado das faces e os estímulos D e E do que os participantes do GUM. Essa hipótese, contudo, precisa ser mais bem investigada.

A combinação do modelo de equivalência de estímulos com o diferencial semântico tem sido usada para investigar o efeito de diferentes parâmetros experimentais sobre a transferência de significados entre estímulos equivalentes. Bortoloti e de Rose (2009) obtiveram indicações de que o MTS atrasado estabelece níveis maiores de transferência de significado do que o MTS simultâneo, o que sugere um grau de relacionamento maior entre os estímulos de classes geradas por MTS atrasado. Barnes-Holmes et al. (2005) mostraram que tentativas de decisão léxica podem detectar o efeito de dica semântica em diferentes níveis com estímulos de uma mesma classe, o que indica que essa metodologia também pode ser utilizada na verificação de variações do grau de relacionamento de estímulos equivalentes. Considerando que o presente estudo empregou somente o MTS simultâneo na geração de classes de equivalência, seria interessante investigar se o MTS atrasado poderia gerar um efeito de dica semântica em níveis mais elevados do que aqueles apresentados aqui.

\section{Referências}

Balota, D. A., \& Lorch, R. F., Jr. (1986). Depth of automatic spreading activation: Mediated priming effects in pronunciation but not in lexical decision. Journal of Experimental Psychology: Learning, Memory, and Cognition, 12(3), 336345.

Barnes-Holmes, D., Staunton, C., Whelan, R., Barnes-Holmes, Y., Commins, S., Walsh, D., et al. (2005). Derived stimulus relations, semantic priming, and event-related potentials: Testing a behavioral theory of semantic networks. Journal of the Experimental Analysis of Behavior, 84(3), 417-433.
Bortoloti, R. (2007). Investigação de propriedades quantitativas de relações simbólicas em quatro estudos experimentais envolvendo o paradigma de equivalência de estímulos. Tese de Doutorado não-publicada, Universidade Federal do Pará, Belém, PA.

Bortoloti, R., \& de Rose, J. C. (2007). Medida do grau de relacionamento entre estímulos equivalentes. Psicologia: Reflexão e Crítica, 20(2), 250-256.

Bortoloti, R., \& de Rose, J. C. (2009). Assessment of the relatedness of equivalent stimuli through a semantic differential. The Psychological Record, 59, 563-590.

Bush, K. M., Sidman, M., \& de Rose, T. (1989). Contextual control of emergent equivalence relations. Journal of the Experimental Analysis of Behavior, 51(1), 29-45.

de Rose, J. C., \& Bortoloti, R. (2007). A equivalência de estímulos como modelo do significado. Acta Comportamentalia, 15(3), 83-102.

de Rose, J. C., McIlvane, W. J., Dube, W. V., Galpin, V. C., \& Stoddard, L. T. (1988). Emergent simple discriminations established by indirect relations to differential consequences. Journal of the Experimental Analysis of Behavior, 50(1), 1-20.

Dimberg, U., Thunberg, M., \& Elmehed, K. (2000). Unconscious facial reactions to emotional facial expressions. Psychological Science, 11(1), 86-89.

Dougher, M., Augustson, E., Markham, M., Greenway, D., \& Wulfert, E. (1994). The transfer of respondent eliciting and extinction functions through stimulus equivalence classes. Journal of the Experimental Analysis of Behavior, 62(3), 331351.

Dube, W., \& Hiris, J. (1997). Matching to Sample Program (Version 11.08) [Computer software]. Waltham, MA: E. K. Shriver Center for Mental Retardation.

Esteves, F., Dimberg, U., \& Öhman, A. (1994). Automatically elicited fear: Conditioned skin conductance responses to masked facial expressions. Cognition and Emotion, 8(5), 383413.

Fields, L., Adams, B. J., Verhave, T., \& Newman, S. (1993). Are stimuli in equivalence classes equally related to each other? The Psychological Record, 43(1), 85-105.

Fields, L., Matneja, P., Varelas, A., Belanich, J., Fitzer, A., \& Shamoun, K. (2002). The formation of linked perceptual classes. Journal of the Experimental Analysis of Behavior, 78(3), 271-290.

Fields, L., Reeve, K. E., Matneja, P., Varelas, A., Belanich, J., Fitzer, A., et al. (2002). The formation of a generalized categorization repertoire: Effect of training with multiple domains, samples, and comparisons. Journal of the Experimental Analysis of Behavior, 78(3), 291-313.

Hayes, S. C., Kohlenberg, B. S., \& Hayes, L. J. (1991). The transfer of contextual control over equivalence classes through equivalence classes: A possible model of social stereotyping. Journal of the Experimental Analysis of Behavior, 56(3), 505-518.

Meyer, D. E., \& Schvaneveldt, R. W. (1971). Facilitation in recognizing pairs of words: Evidence of a dependence between retrieval operations. Journal of Experimental Psychology, 90(2), 227-234.

Morris, J. S., Öhman, A., \& Dolan, R. J. (1998). Conscious and unconscious emotional learning in the amygdala. Nature, 393(6684), 467-470. 
Bortoloti, R. \& Rose, J. C. C. (2011). Avaliação do Efeito de Dica Semântica e da Indução de Significado entre Estímulos Abstratos Equivalentes.

Neely, J. H. (1991). Semantic priming effects in visual word recognition: A selective review of current findings and theories. In D. Besner \& G. W. Humphreys (Eds.), Basic processing in reading: Visual word recognition (pp. 264-336). Hillsdale, NJ: Erlbaum.

Öhman, A. (2002). Automaticity and the amygdala: Nonconscious responses to emotional faces. Current Directions in Psychological Science, 11(2), 62-66.

Osgood, C. E., Suci, G. I., \& Tannenbaum, P. H. (1957). The measurement of meaning. Urbana, IL: University of Illinois Press.

Saunders, R. R., \& Green, G. (1999). A discrimination analysis of training-structure effects on stimulus equivalence outcomes. Journal of the Experimental Analysis of Behavior, 72(1), 117-137.

Saunders, R. R., Saunders, K. J., Kirby, K. C., \& Spradlin, J. E. (1988). The merger and development of equivalence classes by unreinforced conditional selection of comparison stimuli. Journal of the Experimental Analysis of Behavior, 50(2), 145162.

Sidman, M. (1994). Equivalence relations and behavior: A research story. Boston: Authors Cooperative.

Sidman, M., \& Tailby, W. (1982). Conditional discrimination vs. matching-to-sample: An expansion of the testing paradigm. Journal of the Experimental Analysis of Behavior, 37(1), 261-273.

Weisbrod, M., Kiefer, M., Winkler, S., Maier, S., Hill, R., Roesch-Ely, D., et al. (1999). Electrophysiological correlates of direct versus indirect semantic priming in normal volunteers. Cognitive Bain Research, 8(3), 289-298.

Wulfert, E., \& Hayes, S. C. (1988). Transfer of a conditional ordering response through conditional equivalence classes. Journal of the Experimental Analysis of Behavior, 50(2), 125-144. 